\title{
Avaliação do projeto tecnologias sociais para gestão da água mediante sistema de indicadores socioambientais
}

Tecnologias Sociais promovem, por meio de técnicas, processos ou produtos, a transformação social de agentes envolvidos e a melhoria da qualidade de vida. O Projeto Tecnologias Sociais para a Gestão da Água - TSGA, coordenado pela Universidade Federal de Santa Catarina, em parceria com outras instituições catarinense e o Programa Petrobras Ambiental, atuou, por uma década, disseminando boas práticas de gestão de recursos hídricos a comunidades que produzem alimento ou que carecem de saneamento básico rural. Nesse contexto, uma avaliação contínua mostra-se fundamental para garantir o cumprimento dos objetivos e metas propostos e para indicação dos resultados e impactos gerados. O acompanhamento durante o Projeto TSGA era realizado através do Monitoramento e Avaliação do Investimento Ambiental, onde se apresentava o Relatório de Evidências da Matriz Lógica contendo todas as ações desenvolvidas. No entanto, esse documento não avaliou de fato a efetividade e eficácia do Projeto TSGA, tampouco sua abrangência social e o empoderamento do conhecimento pelas comunidades No entanto, esse documento não avaliou de fato a efetividade e eficácia do Projeto TSGA, tampouco sua abrangência social e o empoderamento do conhecimento pelas comunidades TSGA. Indicadores são ferramentas que podem auxiliar a traduzir o significado social e ambiental de projetos visto que detalham, quali ou quantitativamente, os dados dotados de significado social substantivo, usado para substituir, quantificar ou operacionalizar um conceito social abstrato, de interesse coletivo e difuso. Assim, o objeto deste trabalho foi avaliar o Projeto TSGA por meio da proposição de um sistema de indicadores socioambientais que resultou em uma efetividade geral de $71 \%$, considerada satisfatória e em concordância com os aspectos essenciais de uma experiência de tecnologia social. Essa metodologia possibilitou uma compreensão mais concreta das transformaç̃̃es proporcionada, e considerou toda multidimensionalidade do Projeto TSGA, permitindo uma visão ampla e participativa. Verificou-se, portanto, que o Projeto TSGA cumpriu com seus objetivos, princípios e programas e teve o poder de transformar todos os atores sociais envolvidos.

\section{Evaluation of the social technologies for water management project through a system of social and environmental indicators}

\begin{abstract}
Social Technologies promote, through techniques, processes or products, the social transformation of agents involved and the improvement of quality of life. The Social Technologies Project for Water Management - TSGA, coordinated by the Federal University of Santa Catarina, in partnership with other institutions from Santa Catarina and the Petrobras Ambiental Program, has been working for a decade to disseminate good water resources management practices to communities that produce food or who lack rural basic sanitation. In this context, a continuous evaluation is fundamental to ensure compliance with the proposed objectives and targets and to indicate the results and impacts generated. The follow-up during the TSGA Project was carried out through Environmental Investment Monitoring and Assessment, which presented the Logical Matrix Evidence Report containing all actions developed. However, this document did not really assess the effectiveness and effectiveness of the TSGA Project, nor its social coverage and the empowerment of knowledge by the communities involved. There was a need to evaluate in other ways the scope and performance, as well as the legacies left with the exchange of experiences promoted by the TSGA Project actors. Indicators are tools that can help to translate the social and environmed operation lize an abstact social concet of collective and diffuse in operation socioenvion methodology enabled a more concrete understanding of the transformations provided, and considered all the multidimensionality of the TSGA Project, allowing a broad and participatory view. It was verified, therefore, that the Project TSGA fulfilled with its objectives, principles and programs and had the power to transform all the social actors involved.
\end{abstract}

Keywords: Social Technologies; Water Management; Indicators; Evaluation.

Topic: Engenharia da Sustentabilidade e Meio Ambiente

Reviewed anonymously in the process of blind peer.

Luíza Kaschny Borges Burgardt

Universidade Federal de Santa Catarina, Brasil

http://lattes.cnpq.br/9212644455977294

luizakborges@gmail.com

Paulo Belli Filho (iD)

Universidade Federal de Santa Catarina, Brasil

http://lattes.cnpq.br/3239996547346125

http://orcid.org/0000-0003-4338-2830

paulo.belli@ufsc.br

Sérgio Roberto Martins

Universidade Federal de Santa Catarina, Brasil

http://lattes.cnpq.br/8070028111753603

sergiomartins51@gmail.com

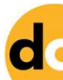

DOI: 10.6008/CBPC2179-6858.2018.007.0017
Received: 10/08/2018

Approved: 24/09/2018
Referencing this:

BURGARDT, L. K. B.; BELLI FILHO, P.; MARTINS, S. R.. Avaliação do projeto tecnologias sociais para gestão da água mediante sistema de indicadores socioambientais. Revista Ibero Americana de Ciências Ambientais, v.9, n.7, p.188-201, 2018. DOI:

http://doi.org/10.6008/CBPC2179-6858.2018.007.0017 


\section{INTRODUÇÃO}

Durante uma década, entre 2007 e 2017, o Projeto Tecnologias Sociais para a Gestão da Água - TSGA, coordenado pela Universidade Federal de Santa Catarina, em parceria com a Empresa de Pesquisa Agropecuária e Extensão Rural de Santa Catarina, o Centro Nacional de Pesquisas em Suínos e Aves da Empresa Brasileira de Pesquisa Agropecuária e o Programa Petrobras Ambiental, trabalhou no diagnóstico, avaliação, tratamento, prevenção e redução da poluição hídrica, bem como na disseminação de modelos e estratégias de conservação da natureza com participação social.

O Projeto TSGA teve seus fundamentos na ideia da governança da água e se baseio nos pressupostos de economia da experiência, comunidade de aprendizagem e gestão local, de modo que buscou integrar as tecnologias e metodologias desenvolvidas pelas instituições proponentes, na implementação de projetos locais; assim como buscou inserir pedagogicamente a comunidade local para o uso das tecnologias e metodologias, visando o empoderamento da comunidade, aumentando a capacidade de gestão local (BAUER, 2010). Por meio do uso de Tecnologia Socais e conscientização e educação ambiental, esse Projeto esteve presente em regiões hidrográficas do Estado de Santa Catarina, disseminando boas práticas de gestão de recursos hídricos, como as bacias hidrográficas do Rio Araranguá, Rio Uruguai e o Aquífero Guarani, atingindo diretamente mais 800 pessoas visto que interviu em conflitos socioambientais presentes na matriz produtiva do estado (agricultura familiar, rizicultura e suinocultura), na preservação e proteção dos mananciais hídricos, assim como no saneamento rural.

Desta forma, como resultados nessas localidades, o Projeto TSGA promoveu a implantação de unidades demonstrativas de Tecnologias Sociais para a gestão da água abordando temas como aproveitamento de água pluvial, recuperação de mata ciliar, sustentabilidade na suinocultura, potabilização alternativa de água, tratamento de efluentes, turismo rural sustentável, e sustentabilidade na rizicultura. Ainda, atuou na formação e capacitação de técnicos, gestores e atores sociais municipais para a gestão do recurso água, ministrando cursos relacionados à temas como gestão social de bacias hidrográficas, recuperação de mata ciliar, saneamento rural, manejo para a qualidade do solo, educação para prevenção e redução de riscos climáticos, uso de geotecnologias livres para apoio à gestão de bacias hidrográficas, monitoramento e diagnóstico de qualidade de água superficial, e geoconservação, geoturismo e geoparques. Também trabalhou na inserção comunitária, com estratégias de educação ambientais para a gestão da água, agindo no contexto escolar com conceitos relacionados à sustentabilidade. Presente em diversas outras ações, o Projeto TSGA promoveu a participação de diversos eventos de caráter científico e pedagógico, visando divulgar os resultados e tecnologias sociais implementadas, além de conscientizar, formar a capacitar alunos, professores e a própria comunidade para a importância do bom uso e gestão da água.

A entidade financiadora do Projeto TSGA, para acompanhamento e avaliação das ações desenvolvidas pela entidade executora, solicitava a apresentação de um Relatório de Evidências da Matriz Lógica - MAIA. A Matriz Lógica é uma ferramenta que propicia a visualização clara do planejamento do projeto, incluindo os mecanismos de avaliação de resultados. No entanto, a avaliação do Projeto TSGA por 
meio dos MAIAs é descritiva e quantitativa, visto que relaciona apenas os trabalhos realizados durante o Projeto, e seus custos, para alcance de todos seus objetivos. Esse documento não avalia de fato a efetividade e eficácia do Projeto, tampouco sua abrangência social e o empoderamento do conhecimento adquirido pelas comunidades envolvidas. Muito mais do contar quantas tecnologias sociais foram implementadas ou quantos cursos de capacitação foram realizados ou quanto custou cada ação do Projeto, há necessidade de se avaliar os efeitos que o Projeto trouxe para comunidades e os atores sociais. Desta forma, este trabalho visa analisar de outras maneiras a abrangência e o desempenho, assim como os legados deixados com a troca de experiências promovidas pelo stakeholders do Projeto TSGA.

Diversos são os métodos de avaliação de projetos de cunho social e ambiental, porém, destaca-se a avaliação de impacto, que se refere aos impactos sociais e ambientais que os objetivos propostos causaram na área do projeto, e às transformações comportamentais percebidas no público-alvo e/ou na comunidade. A avaliação de impacto exprime os resultados e feitos da intervenção de determinada ação, mesmo após seu término, sendo que sua intenção é determinar mais amplamente se o programa teve os efeitos desejados nos indivíduos ou instituições e se aqueles efeitos podem ser atribuídos à intervenção do programa (OLIVEIRA, 2013). Assim, indicadores são ferramentas que podem auxiliar a traduzir o significado social e ambiental de projetos visto que detalham, quali ou quantitativamente, os dados dotados de significado social substantivo, usado para substituir, quantificar ou operacionalizar um conceito social abstrato, de interesse difuso.

Portanto, o objetivo deste trabalho foi avaliar o Projeto Tecnologias Sociais para Gestão da Água, desenvolvido em cinco regiões do estado de Santa Catarina, de forma holística, pluralista e abrangente, envolvendo principalmente, os impactos gerados nos atores sociais, mediante o uso de um sistema de indicadores socioambientais, em uma perspectiva sustentável, relacionando o grau de empoderamento e de transformação social da comunidade envolvida para com a gestão da água.

\section{REVISÃO TEÓRICA}

As tecnologias desempenham um papel central nos processos de mudança social na medida em que a resolução da problemática da pobreza, do desemprego, da exclusão social e do subdesenvolvimento não pode ser analisada sem levar em conta a dimensão tecnológica (THOMAS, 2009). Assim, dentre os muitos modelos tecnológicos disponíveis, a reversão desse quadro depende da incorporação de novos conceitos e valores no processo decisório, o que implica repensar o próprio processo de desenvolvimento tecnológico a partir de uma nova ética centrada nos valores de igualdade e solidariedade em conjunto com as questões econômicas (DAGNINO, 2004).

No entanto, tais tecnologias só poderão incorporar esses novos valores se constituírem num elemento eficaz para a consecução de um desenvolvimento que seja efetivamente sustentável se forem socialmente construídas, pois a inovação não pode ser pensada como algo feito num lugar e aplicada em outro, mas como um processo desenvolvido no lugar e pelos atores sociais que efetivamente lhe farão uso (ARAÚJO et al., 2017). Requer-se, então, a superação dessa visão tradicional de tecnologia pronta, baseada 
apenas em requisitos técnico-econômicos, desenvolvida de forma desvinculada da realidade e das capacidades cognitivas dos atores sociais que efetivamente lhe farão uso, com a consequente incorporação dos aspectos sociais e ambientais que constituem a relação Ciência, Tecnologia e Sociedade (LASSANCE et al., 2004).

Desta forma, a Tecnologia Social vem sendo discutida no Brasil no início da primeira década do século XXI por diferentes atores sociais (organizações da sociedade civil, universidades, integrantes do governo, trabalhadores) após a preocupação trazida pela Constituição Federal de 1988 para promoção e incentivo do progresso da ciência, tecnologia e inovação visando o desenvolvimento do sistema produtivo, cultural e socioeconômico, visto a crescente exclusão social e precarização do trabalho (FERNANDES et al., 2010). A Tecnologia Social vem se constituindo como uma das respostas possíveis para o atendimento das demandas sociais, cujo objetivo é o de responder à problemática de desenvolvimento comunitário, promovendo a inclusão social com a geração de renda e de alternativas tecno-produtivas, em cenários socioeconômicos caracterizados por situações de extrema pobreza (THOMAS, 2009).

O movimento da Tecnologia Social (TS) parte desse princípio para desenvolver tecnologias que incorporem, desde a sua concepção até a aplicação, monitoramento e avaliação, uma intencionalidade de inclusão social e de um desenvolvimento social, econômico e ambiental sustentável, devendo ser definida de acordo com o contexto, pela relação particular entre a tecnologia e a sociedade e o envolvimento dos atores interessados (FONSECA, 2010). Diversos estudos têm evidenciado essa capacidade das tecnologias sociais para contribuir com a consecução desse novo modelo de desenvolvimento mais justo e solidário, assim, observa-se a crescente adoção desse tipo de tecnologia em projetos de desenvolvimento, seja de iniciativa pública ou privada, sobretudo daqueles espaços geográficos aonde residem as parcelas mais pobres da população (ARAÚJO et al., 2017).

Nesse sentido, em 2004, o Instituto de Tecnologias Sociais (ITS) desenvolveu o conceito de Tecnologia Social definindo-a como sendo o conjunto de técnicas, metodologias transformadores, desenvolvidas e/ou aplicadas na interação com a população e apropriada por ela, que representam soluções para inclusão social e melhoria das condições de vida. Para Irma Passoni, uma das fundadoras do Instituto, a Tecnologia Social é a ferramenta que agrega informação e conhecimento para mudar a realidade, servindo como uma ponte entre as necessidades, os problemas e as soluções (ITS, 2004).

Pode-se afirmar que as Tecnologias Sociais promovem a transformação social, visto que estão mais sintonizadas com as demandas da sociedade para promover um desenvolvimento participativo e de inclusão social (OLIVEIRA et al., 2012). Desta forma, as experiências com Tecnologias Sociais devem ser monitoradas e avaliadas por meio de uma metodologia que contempla toda a complexidade, multidimensionalidade e interdisciplinaridade das Tecnologias Sociais da maneira mais harmônica e equilibrada possível.

Essa avaliação pode ocorrer de diversas formas e pode variar de acordo com as exigências do agente financiador ou dos entes apoiadores. Avaliação de impacto, por exemplo, refere-se aos impactos sociais e ambientais que os objetivos propostos causaram na área do projeto, e às transformações comportamentais percebidas no público-alvo e/ou na comunidade. Ainda, constitui-se no exame da relação entre o esforço 
empregado na implementação e os resultados obtidos, mas se diferencia da efetividade por ser mais ampla e complexa, visto que capta os efeitos do programa e a sua relação com a intenção da política, e as alterações efetivas nos beneficiários. Portanto, é aquela que revela o desempenho da ação com propósito de mudança (STEPHANOU, 2005). No entanto, a avaliação de impacto representa um desafio, uma vez que os ganhos obtidos não são facilmente medidos, pois se referem a questões culturais, à mudança de valores e novas atitudes, mensurando a contribuição do projeto para a emancipação das comunidades atingidas e sua mais eficiente organização e atuação política (LAZZARINI et al., 2015).

Assim, dependendo do objetivo da avaliação de um projeto, os indicadores são referências quantitativas ou qualitativas que servem para indicar se as atividades do projeto estão sendo executadas (indicadores de processo ou de desempenho) ou se os objetivos foram alcançados (indicadores de resultado ou de impacto). Desta forma, os processos avaliativos precisam apresentar uma abordagem pluralista, que envolva aspectos qualitativos e quantitativos e considere os diferentes stakeholders envolvidos (VALARELLI, 1999). Os processos são enriquecidos quando há o envolvimento de diferentes atores e a preocupação em se criar um sistema diversificado de indicadores, combinando conceitos, meios de coleta e responsáveis diferentes.

Coelho (2004) acredita que indicadores são ferramentas que permitem a obtenção de informações sobre uma dada realidade e que possuem como principal característica a de poder sintetizar um conjunto complexo de informações, retendo apenas o significado essencial dos aspectos analisados. Valarelli (1999) afirma que são parâmetros qualificados e/ou quantificados que servem para detalhar em que medida os objetivos de um projeto foram alcançados, dentro de um prazo delimitado de tempo e numa localidade específica, principalmente em contextos sociais.

Para autores como Silva et al. (2012), os indicadores têm como principal uso o monitoramento da alteração de comportamentos num sistema, constituindo-se como o instrumento de acompanhamento dessas mudanças, dando informações sobre o presente estado e evolução do sistema. Assim como para a OCDE (2004) que se refere a monitoramento como um processo de acompanhamento e avaliação dos desempenhos e progressos. Ainda, autores como OCDE (2004) e Silva et al. (2012) acreditam que os indicadores também tem como objetivo o planejamento dos projetos e a produção da informação.

A proposição de um instrumento que possibilite a realização de diagnóstico, monitoramento e avaliação de projetos socioambientais representaria um avanço considerável no campo analítico, ao tempo em que geraria o substrato qualitativo para as avaliações de projetos baseados em tecnologias sociais (VALARELLI, 1999). Atualmente, há um rico processo no qual muitas organizações admitem a necessidade e se percebem motivadas a desenvolver um sistema de indicadores de resultados que thes seja adequado para ter condições de avaliar até que ponto os objetivos dos seus projetos estão sendo alcançados (ARAÚJO et al., 2017).

O estabelecimento de um sistema de indicadores é fundamental para realizar uma análise holística do projeto socioambiental visto que abranja desde o processo de construção e difusão até os resultados e 
impactos gerados pela experiência de tecnologia social, possibilitando indicar o nível de sucesso da experiência e monitorar e avaliar os resultados e impactos gerados a partir da sua adoção (MINAYO, 2009).

\section{METODOLOGIA}

Assim como foi definido o método da Matriz Lógica pelo financiador do Projeto Tecnologias Socais para Gestão da Água para avaliação de resultados, os quais expressam os benefícios diretos ou indiretos das ações empreendidas, este trabalho utilizou indicadores de impactos para identificar os efeitos socioambientais do Projeto TSGA considerando seus objetivos e aspectos como organização e participação social, governança e gestão da água, melhoria da qualidade de vida, inclusão social, apropriação e aprendizagem, empoderamento, capacitação e formação.

Portanto, considerando toda a revisão da literatura quanto aos métodos de avaliação de projetos socioambientais, foi realizada uma adaptação do Sistema de Indicadores para Diagnóstico, Monitoramento e Avaliação de Tecnologias Sociais (SIDMATECS), proposto por Araújo (2015), o qual desenvolveu um método de avaliação de projetos e programas que utilizam tecnologias sociais para promoção da melhoria da qualidade vida das comunidades envolvidas, contemplando toda a complexidade, multidimensionalidade e interdisciplinaridade das Tecnologias Sociais.

O autor aponta que os projetos que trabalham com tecnologias sociais passam primeiramente pelas etapas de construção e difusão, para posteriormente gerarem resultados e impactos. Relativamente à fase de construção e difusão, os programas, experiências e atividades são construídos segundo um fluxo de planejamento, desenvolvimento e aplicação, para posterior difusão por meio da reaplicação (ARAÚJO, 2015). Somente com o processo de reaplicação é que surge o empoderamento e a apropriação do conhecimento.

Para cada uma das etapas das fases de construção e difusão e de resultados e impactos, o autor levantou na literatura o maior número possível de indicadores, totalizando um número de cento e trinta. No entanto, acredita ser inviável a aplicação em sua totalidade e sugere a adoção de três a quatro indicadores por fase, variando entre vinte e quatro e trinta e dois indicadores no total.

Nesse sentido, para a avaliação do Projeto Tecnologias Sociais para a Gestão da Água, foi proposto utilizar as quatro etapas da fase de construção e difusão do SIDMATECS de Araújo (2015): planejamento, desenvolvimento, aplicação e reaplicação. Assim como o autor, identificou-se que essas quatro etapas são necessárias para que os resultados e impactos do Projeto TSGA ocorram no processo de transformação social dos atores envolvidos. Ainda, foi proposto adaptar a fase de resultados e impactos do SIDMATECS de Araújo (2015), com as etapas de impactos e empoderamento, as quais estão relacionadas à inclusão social e qualidade de vida, bem como apropriação do conhecimento para reaplicação das experiências.

Desta forma, os indicadores selecionados para esta pesquisa foram: Etapa de planejamento: Atores sociais locais consultados previamente ao projeto; Grau de inovação; Vinculação a uma demanda social concreta; Conservação dos recursos naturais envolvidos; Etapa de desenvolvimento: Respeito às identidades locais; Mobilização da população; Valorização do conhecimento popular; Geração e obtenção de novos conhecimentos; Conscientização sobre questões ambientais; Etapa de aplicação: Participação dos usuários 
nas etapas do processo; Desenvolvimento de novas habilidades; Apoio institucional; Disponibilidade local dos insumos necessários; Etapa de reaplicação: Aperfeiçoamento contínuo das soluções; Baixo custo de implantação e manutenção; Domínio público do conhecimento; Capacidade de disseminação pelos usuários; Etapa de impactos: Conservação e manutenção dos recursos hídricos; Inclusão social; Melhoria na qualidade de vida; Acesso ao saneamento básico; Apoio social; Etapa de empoderamento: Capacidade de realização; Aquisição de novos conhecimentos; Transformação social; Construção de espaços para discussão; Trabalho em rede.

Diversas técnicas de coleta de dados podem ser aplicadas para a avaliação de projetos, no caso de Tecnologias Sociais, a participação dos atores sociais e institucionais é muito forte na sua construção, sendo, portanto, imprescindível na hora da avaliação. Desta forma, a análise das experiências promovidas pelo Projeto Tecnologias Sociais para a Gestão da Água foi realizada por meio da aplicação de questionários online aos atores sociais como forma de coleta de dados, contemplando as questões relacionadas aos indicadores estabelecidos. Para os atores sociais que foram diretamente beneficiados pelo Projeto TSGA foi realizada uma visita presencial para, além da coleta de dados por meio da aplicação do questionário, ter o conhecimento prático das ações realizadas no âmbito do Projeto TSGA. Essas visitas também permitiram uma visão ampla e participativa no processo de conversação, onde todas as intervenções e comentários foram considerados, visto que possibilitou a liberdade de expressão ao entrevistado.

Os respondentes puderam expressar sua opinião por meio de uma escala quantitativa que variou de acordo com seu grau de aceitação em relação às afirmativas expostas. Para cada pergunta do questionário, foi obtido um resultado quantitativo, conforme a seguinte conversão (quadro 1), considerando sempre o caráter participativo e contemplando a percepção dos atores sociais, no caso das visitas.

Quadro 1: Conversão do resultado.

\begin{tabular}{|l|l|l|}
\hline Escala qualitativa & Escala quantitativa \\
\hline Não satisfatório & Não ou discordo totalmente & 1 \\
\hline Parcialmente satisfatório & Aceito ou concordo & 2 \\
\hline Satisfatório & Sim ou concordo totalmente & 3 \\
\hline
\end{tabular}

O Sistema de Indicadores para Diagnóstico, Monitoramento e Avaliação de Tecnologias Sociais (SIDMATECS), proposto por Araujo (2015) constitui-se em uma ferramenta de representação do estado de uma determinada experiência tecnológica por meio de um índice sintético que representa o seu nível de convergência com os fundamentos da tecnologia social. No caso do Projeto TSGA, além da questão das tecnologias sociais, outras ações foram desenvolvidas como programas de formação e capacitação, inserção comunitária e educação ambiental.

Desta forma, para complementar os resultados da coleta de dados foi aplicada uma pequena análise estatística, na qual se buscou, para cada indicador, a média aritmética, desvio padrão e coeficiente de variação. A média aritmética é uma medida de centralidade que representa o valor de concentração dos dados de uma determinada distribuição, sendo o resultado da soma dos elementos de um conjunto de dados dividida pela quantidade de elementos total. Já o desvio padrão, é uma medida de dispersão dos dados em torno da média, sendo que um valor baixo e desvio padrão indica que os dados tentem a estarem próximos 
da média; enquanto que um valor alto indica que os dados estão espalhados por uma ampla gama de valores. Por fim, o coeficiente de variação é uma medida padronizada de dispersão de uma distribuição e mostra a extensão da variabilidade dos dados em relação à média. Ainda, como os indicadores tiveram uma escala de variação entre 1 e 3 , foram convertidos em índices variando de 0 a 1.

\section{RESULTADOS E DISCUSSÃO}

A avaliação proposta para o Projeto Tecnologias Sociais para Gestão da Água teve seu desempenho baseado nas seis etapas descritas anteriormente, sendo fruto da agregação dos índices obtidos em cada um delas, conforme resultado apresentado no quadro 2 abaixo. 0 objetivo dessa avaliação foi verificar a efetividade do Projeto TSGA em uma perspectiva sustentável, avaliando, principalmente, os impactos social e ambiental conforme a visão de todos os participantes (gestores, executores e beneficiados).

Quadro 2: Resultado da avaliação do Projeto TSGA.

\begin{tabular}{|l|l|l|l|l|l|}
\hline Etapas de avaliação & Média & Desvio padrão & Coeficiente de variação (\%) & Índice por etapa & Índice total \\
\hline Planejamento & 2,478 & 0,533 & 21,78 & 0,73 & 0,71 \\
\hline Desenvolvimento & 2,567 & 0,510 & 20,05 & 0,78 \\
\hline Aplicação & 2,296 & 0,623 & 27,40 & 0,63 \\
\hline Reaplicação & 2,397 & 0,561 & 23,85 & 0,69 \\
\hline Impactos & 2,394 & 0,584 & 24,55 & 0,70 & \\
\hline Empoderamento & 2,446 & 0,572 & 20,78 & 0,72 & \\
\hline
\end{tabular}

Considerando que esta análise é holística e o Projeto TSGA teve diversas frentes de abrangência, esta avaliação resultou em uma efetividade de $71 \%$, com maior contribuição pela etapa de desenvolvimento (78\%); e menor, pela etapa de aplicação (63\%), que em nada impediu resultados satisfatórios para as etapas de impactos e empoderamento, sendo esses dois últimos considerados os efeitos e legados que o Projeto TSGA proporcionou aos atores envolvidos. Quanto à dispersão da amostra, a etapa de desenvolvimento obteve menor resultado, com 0,510 de desvio padrão e a etapa de aplicação o maior, com 0,623 . A variabilidade dos resultados foi de 20,05\% (etapa de desenvolvimento) a 27,40\% (etapa de aplicação).

A etapa de planejamento do Projeto TSGA foi considerada no âmbito desta avaliação por estar relacionada com a preparação, organização e estruturação das ações que foram desenvolvidas para atingir os resultados de cada um dos objetivos específicos do Projeto TSGA. Para tanto, foram considerados quatro indicadores: (1) Atores sociais locais consultados previamente ao Projeto, para verificação do nível de participação social dos atores envolvidos nesta etapa; (2) Grau de inovação, para verificação da percepção dos atores sociais sobre a inovação das experiências promovidas pelo Projeto; (3) Vinculação a uma demanda social concreta, para verificação da percepção dos atores sociais acerca da capacidade do Projeto em atender a uma demanda social da comunidade; e (4) Conservação dos recursos naturais envolvidos, para verificação da percepção dos atores sociais em relação ao potencial do Projeto na gestão da água e preservação dos recursos naturais. Com base na avaliação realizada por todos os atores envolvidos no Projeto TSGA (gestores, executores e beneficiados), o índice obtido na etapa de planejamento foi de 0,73.

Considerando que o planejamento é a gênese de todo o projeto, visto que as demandas a serem trabalhadas e suas possíveis soluções devem ser identificadas; e considerando a abrangência do Projeto 
TSGA, tanto em termos de comunidade envolvida, quanto em termos de perpetuação ao longo do tempo, a efetividade de $73 \%$ dos respondentes para a etapa de planejamento é considerada satisfatória e convergente com os pressupostos do Projeto e do conceito de tecnologias sociais. O Projeto TSGA, conforme descrito anteriormente, se baseou nos fundamentos de governança da água, economia de experiência, comunidade de aprendizagem e gestão local para o fortalecimento do uso sustentável da água por meio do apoio à capacitação e disseminação do conhecimento, assim como da implementação de unidades demonstrativas de tecnologias sociais. Por sua vez, o conceito de tecnologias sociais está relacionado aos métodos desenvolvidos na interação com a comunidade para execução de efetivas soluções de transformação social. Assim, devem ser desenvolvidas e praticadas na interação com a população, e apropriada por ela, obedecendo à princípios como a ideia da tecnologia ser construída para atender demandas concretas da população em um processo que envolva os interessados. Desta forma, novos conhecimentos podem ser construídos pelo conjunto de atores envolvidos em um processo democrático e participativo, planejado e sistematizado, visando a sustentabilidade (CAVALCANTI et al., 2011). Assim, os quatro indicadores escolhidos para a etapa de planejamento tiveram o propósito de avaliar o nível de participação social na elaboração de uma ação efetiva relacionada à uma demanda concreta, ou seja, à conservação e preservação dos recursos naturais, especificamente relacionados com a gestão da água e sustentabilidade.

A etapa de desenvolvimento do Projeto TSGA esteve relacionada com o processo de construção das ações a serem implementadas, estando associada à evolução e crescimento das técnicas e métodos desenvolvidos, bem como do conhecimento disseminado relacionado à gestão da água e sustentabilidade. Para tanto, foram considerados cinco indicadores: (1) Respeito às identidades locais, para verificação da percepção dos atores sociais sobre o grau de incorporação da cultura local no Projeto; (2) Mobilização da população, para verificação da percepção dos atores sociais acerca de seu envolvimento na implantação das ações; (3) Valorização do conhecimento popular, para verificação da percepção dos atores sociais quanto à valorização e integração dos conhecimentos populares nas ações do Projeto; (4) Geração e obtenção de novos conhecimentos, para verificação da percepção dos atores sociais sobre a capacidade do Projeto ter proporcionado a geração e apropriação de novos conhecimentos; e (5) Conscientização sobre questões ambientais, para verificação da percepção dos atores sociais sobre a capacidade do Projeto em promover melhorias relacionadas à sustentabilidade ambiental. Com base na avaliação realizada por todos os atores envolvidos no Projeto TSGA (gestores, executores e beneficiados), o índice obtido na etapa de desenvolvimento foi de 0,78 .

Considerando que o Projeto TSGA durou cerca de dez anos, concentrado em basicamente duas fases, e que o desenvolvimento de um projeto de tamanha natureza social e ambiental é dificultoso, ainda mais com as diversas frentes que esse Projeto proporcionou, a efetividade de $78 \%$ dos respondentes para a etapa de desenvolvimento pode ser considerada muito boa e igualmente convergente com seus pressupostos e conceito de tecnologias sociais. Durante a execução do Projeto, para atendimento de seus objetivos, foi preciso que o conhecimento acadêmico se transformasse em ações efetivas para execução de um processo de mudança e transformação com a geração de benefícios para a comunidade envolvida. Desta forma, os 
cinco indicadores escolhidos para a etapa de desenvolvimento tiveram o propósito de avaliar a construção das ações considerando e apreciando as identidades locais para enriquecimento de seus conhecimentos e conscientização da gestão da água.

Diferente da avaliação da etapa de desenvolvimento, que esteve relacionada com o processo de construção das ações a serem realizadas, a etapa de aplicação do Projeto TSGA diz respeito ao usufruto propriamente dito do Projeto, seja ele na questão da implementação das unidades demonstrativas de tecnologias sociais, ou na educação ambiental, ou na capacitação de gestores, por exemplo. Para tanto, foram considerados quatro indicadores: (1) Participação dos usuários nas etapas do processo, para verificação do nível de participação social dos atores envolvidos no Projeto; (2) Desenvolvimento de novas habilidades, para verificação da percepção dos atores sociais quanto ao desenvolvimento de novas habilidades adquiridas pelo Projeto; (3) Apoio institucional, para verificação da percepção dos atores sociais na relação com as instituições proponentes do Projeto; e (4) Disponibilidade local dos insumos necessários, para verificação da percepção dos atores sociais quanto ao uso de insumos locais. Com base na avaliação realizada por todos os atores envolvidos no Projeto TSGA (gestores, executores e beneficiados), o índice obtido na etapa de aplicação foi de 0,63 .

Considerando que a aplicação do Projeto TSGA se deu basicamente por quatro programas estruturantes, nos quais foram implementadas unidades demonstrativas de tecnologias sociais para a gestão da água e foram realizadas ações de formação e capacitação com a inserção da comunidade em estratégias de educação ambiental, sendo priorizada a comunicação e a disseminação da informação, para fortalecimento da sustentabilidade, a avaliação da etapa de aplicação, que neste contexto está relacionada ao ato de usufruir das ações do Projeto, resultou no menor dos índices de todas as etapas, com efetividade de $63 \%$ segundo os respondentes desta pesquisa. Apesar do valor se mostrar abaixo do esperado, acreditase que, pelo fato da abrangência do Projeto, das diversas instituições proponentes e executoras e da dificuldade de persuasão junto às comunidades beneficiadas, a aplicação do Projeto TSGA foi complicada.

A avaliação da etapa de reaplicação está relacionada com o poder de difusão do conhecimento adquirido e possibilidade de ganhos de escala, ou seja, possibilidade de reprodução das informações e técnicas aprendidas, podendo surtir, como consequência, efeito de mudança e transformação nas comunidades beneficiadas. Para tanto, foram considerados quatro indicadores: (1) Aperfeiçoamento contínuo das soluções, para verificação da percepção dos atores sociais quanto à perpetuação das técnicas promovidas pelo Projeto; (2) Baixo custo de implantação e manutenção, para verificação da percepção dos atores sociais quanto aos custos relacionados às condições econômicas e financeiras das comunidades envolvidas; (3) Domínio público do conhecimento, para verificação da percepção dos atores sociais quanto à acessibilidade do conhecimento público adquirido no Projeto; e (4) Capacidade de disseminação pelos usuários, para verificação da percepção dos atores sociais quanto à transmissão do conhecimento adquirido pelo Projeto. Com base na avaliação realizada por todos os atores envolvidos no Projeto TSGA (gestores, executores e beneficiados), o índice obtido na etapa de reaplicação foi de 0,69. 
Considerando que a reaplicação do conhecimento adquirido durante o Projeto TSGA é fator primordial nesta análise, visto que, conforme demonstrado anteriormente, as experiências relacionadas às tecnologias sociais são construídas segundo um fluxo que percorre as etapas de planejamento, desenvolvimento e aplicação para posteriormente ocorrer sua difusão, passando a produzir os resultados e impactos propostos (ARAÚJO, 2015), apesar do índice ter se mostrado abaixo do esperado, a avaliação da etapa de reaplicação resultou na efetividade de $69 \%$, segundo os respondentes desta pesquisa. Ressalta-se que esta etapa pode ser considerada a mais difícil de atingir resultados efetivos, pois a apropriação do conhecimento pelas comunidades beneficiadas não é tarefa fácil, apesar das estratégias delineadas pelo Projeto TSGA para alcance desse objetivo.

Após a fase de construção e difusão, composta pelas etapas de planejamento, desenvolvimento, aplicação e reaplicação, tem-se a fase de resultados, composta pelas etapas de impactos e empoderamento. Assim, a etapa de impactos do Projeto TSGA esteve relacionada com os efeitos e legados produzidos a partir da reaplicação do conhecimento adquirido pelos beneficiados. Para tanto, foram considerados cinco indicadores: (1) Conservação e manutenção dos recursos hídricos, para verificação da percepção dos atores sociais acerca do impacto do Projeto na gestão da água e manutenção dos recursos hídricos; (2) Inclusão social, para verificação da percepção dos atores sociais quanto à inclusão da comunidade impactada pelo Projeto; (3) Melhoria na qualidade de vida, para verificação da percepção dos atores sociais quanto à melhoria na qualidade de vida proporcionada pelo Projeto; (4) Acesso ao saneamento básico, para verificação da percepção dos atores sociais quanto o acesso ao saneamento básico, principalmente relacionado ao abastecimento de água e destinação de efluentes; e (5) Apoio social, para verificação da percepção dos atores sociais em relação ao fortalecimento das comunidades envolvidas no Projeto. Com base na avaliação realizada por todos os atores envolvidos no Projeto TSGA (gestores, executores e beneficiados), o índice obtido na etapa de impactos foi de 0,70.

Considerando que para avaliar os impactos do Projeto TSGA nas comunidades envolvidas foram observados os benefícios gerados em termos de transformação social e ambiental, o resultado da efetividade de $70 \%$ da etapa de impacto é satisfatório e correspondente aos objetivos delineados pelo Projeto. Para que o projeto tenha a capacidade de mudança de comportamento, as estratégias do Projeto se apoiaram fortemente na disseminação do conhecimento e fortalecimento de práticas de uso sustentável dos recursos naturais. Ainda, foi construído o Centro de Tecnologias Sociais para Gestão da Água, estrutura permanente para promoção do desenvolvimento de novos projetos de pesquisa, extensão e ensino relacionados a essa temática, bem como promoção de apoio à formação e capacitação. Desta forma, os indicadores da etapa de impactos estiveram relacionados com os resultados gerados pelo Projeto TSGA, sendo eles a melhoria da gestão dos recursos naturais, e na qualidade de vida, propiciando acesso ao saneamento básico e inclusão social.

Finalizando a fase de resultados, tem-se a etapa de empoderamento do Projeto TSGA que está relacionada com a busca pela autonomia dos atores beneficiados por meio da apropriação do conhecimento de tal forma que sejam capazes de reaplicação. Conforme Oakley et al. (2003, citado por ARAújO, 2015), a 
noção de empoderamento refere-se ao processo de confronto de desequilíbrios de poder ao mesmo tempo que apoio ativamente aos desprovidos de poder para que favorecimento do processo de independência. No contexto de tecnologias sociais, busca-se a autonomia da comunidade que está às margens dos processos de desenvolvimento tradicionais por meio do fortalecimento do conhecimento e alcance de métodos antes inacessíveis, promovendo relacionamentos com outras esferas de poder para conduzir seu próprio processo de desenvolvimento (ARAÚJO, 2015). Para tanto, foram considerados cinco indicadores: (1) Capacidade de realização, para verificação do impacto do Projeto na capacidade de realização dos atores sociais; (2) Aquisição de novos conhecimentos, para verificação da percepção dos atores sociais quanto à aquisição de novos conhecimentos pelo Projeto; (3) Transformação social, para verificação da percepção dos atores sociais quanto às mudanças comportamentais após o Projeto; (4) Construção de espaços para discussão, para verificação da percepção dos atores sociais quanto à construção de novos meios para discussão da comunidade impactada pelo Projeto; e (5) Trabalho em rede, para verificação da percepção dos atores sociais acerca do desenvolvimento de trabalhos em rede. Com base na avaliação realizada por todos os atores envolvidos no Projeto TSGA (gestores, executores e beneficiados), o índice obtido na etapa de empoderamento foi de 0,72 .

Considerando que a etapa de empoderamento somente poderia ser alcançada se todas as etapas anteriores fossem executadas com sucesso, a efetividade de $72 \%$ é bastante positivo para um projeto de tamanha natureza. Para tanto, os indicadores escolhidos tiveram relação com a apropriação do conhecimento disseminado pelo Projeto TSGA às comunidades beneficiadas. No entanto, analisando as manifestações dos respondentes aos questionários, ficou muito claro que não só os atores considerados beneficiados puderam desfrutar dos impactos do Projeto TSGA. Para a equipe TSGA também representou um aprendizado na área tecnológica, comportamental e organizacional, sendo uma experiência rica em saberes.

\section{CONCLUSÕES}

Por meio de um sistema de indicadores socioambientais de avaliação foi possível uma compreensão mais concreta das transformações proporcionadas pelo Projeto Tecnologias Sociais para Gestão da Água, o qual se mostrou um desafio pelo fato de medir o impacto causado nos atores sociais. Essa avaliação permitiu constatar, em todas as fases do Projeto TSGA, a importância do seu caráter sistêmico, participativo e interdisciplinar, fatos que evidenciam ainda mais a complexidade desta pesquisa.

Desta forma, a adaptação ao Sistema de Indicadores para Diagnóstico, Monitoramento e Avaliação de Tecnologias Sociais (SIDMATECS), desenvolvido por Araújo (2015), como instrumento de avaliação do Projeto TSGA se mostrou adequada visto que considera que os projetos que trabalham com tecnologias sociais passam primeiramente pelas etapas de construção e difusão, para posteriormente gerarem resultados e impactos. Para tanto, indicadores socioambientais foram consideradas nas etapas de planejamento, desenvolvimento, aplicação, reaplicação, impactos e empoderamento, sendo as duas últimas referentes aos efeitos e legados produzidos a partir da apropriação e reaplicação do conhecimento adquirido 
pelos atores sociais do Projeto TSGA. Os principais elementos que indicam o impacto e empoderamento nesta pesquisa estiveram relacionados aos aspectos de conservação e manutenção dos recursos hídricos, melhoria da qualidade de vida, acesso ao saneamento básico, capacidade de realização, aquisição de novos conhecimentos, trabalho em rede e transformação social.

Com as visitas nas comunidades beneficiadas foi possível identificar a perpetuação das ações implementadas mesmo após o término do Projeto TSGA visto que o conhecimento e as técnicas adquiridas continuam sendo reaplicados em muitas localidades. Verificou-se que o Projeto TSGA claramente contribuiu para a melhoria das condições de vida e bem-estar dos atores sociais envolvidos, não somente aqueles beneficiados diretamente, mas todos aqueles que de alguma forma participaram e desenvolveram as ações ao longo do tempo, como a comunidade acadêmica.

Como resultado do sistema de avaliação socioambiental identificou-se $71 \%$ de efetividade do Projeto TSGA e em concordância com os aspectos essenciais de uma experiência de tecnologia social. O Projeto impactou positivamente os envolvidos nas questões relacionadas à construção do conhecimento sobre o meio ambiente, gerando inovação e aperfeiçoamento das soluções nas comunidades beneficiadas por meio de um processo pedagógico que envolveu o diálogo entre os saberes para adequada apropriação do conhecimento pelas comunidades. Igualmente, conseguiu mobilizar a população durante o desenvolvimento das atividades por meio de uma participação democrática, respeitando a cultura e as identidades locais e fortalecendo espaços para discussão, gerando um grande trabalho em rede. Desta forma, verificou-se que o Projeto TSGA atingiu seus objetivos visto que propiciou práticas de conservação e manutenção dos recursos hídricos e sustentabilidade do meio rural nas comunidades beneficiadas com a implantação das unidades demonstrativas de tecnologias sociais, Ainda, provocou melhoria na qualidade de vida e transformação social de todos os atores envolvidos os quais se desenvolveram de forma sustentável e comunitária, e de toda comunidade acadêmica que participou as ações do Projeto TSGA.

Ainda, os resultados desta pesquisa também apontaram que os elementos essenciais para a aplicação das ações do Projeto poderiam ter sido melhores utilizados e que o fácil acesso aos insumos, assim como a implantação em termos financeiros, pesou na reaplicação das ações pela comunidade beneficiada. O Projeto TSGA poderia ter fomentado uma metodologia mais participativa de todos os atores sociais durante todas as etapas visando impulsionar a disseminação e reaplicação do conhecimento adquirido. Porém, considerando que esta análise é holística e o Projeto TSGA teve diversas frentes de abrangência, esses fatores não impediram resultados satisfatórios relacionados aos impactos e empoderamento, sendo esses dois últimos considerados os efeitos e legados que o Projeto TSGA proporcionou aos atores envolvidos.

Ressalta-se que o Projeto TSGA foi uma oportunidade de desenvolvimento tecnológico associado com apoio pedagógico e de valorização do conhecimento local para a sustentabilidade, tanto para as instituições, como para as comunidades envolvidas. O Projeto TSGA foi um divisor de águas na vida de muitas pessoas, que enxergaram nas tecnologias sociais a possibilidade de atuar em uma área que envolva as questões sociais, econômicas, ambientais e culturais. Além destes, transformou a vida de produtores, de comunidades e de pessoas que vivenciam no dia-a-dia as dificuldades na oferta de água de qualidade, e que 
puderam - a partir dos conhecimentos repassados - modificar as suas realidades aplicando e replicando tecnologias acessíveis.

Por fim, ressalta-se que o Projeto TSGA foi um marco importante para a gestão dos recursos hídricos no Estado de Santa Catarina visto que envolveu a colaboração de diversas instituições, com a formação de redes colaborativas, cujas parcerias continuam atuantes, tendo caráter pioneiro e inovador sendo sua atitude protagonista no envolvimento e empoderamento social com o viés educacional.

\section{REFERÊNCIAS}

ARAÚJO, R. O. A.. Tecnologias sociais e suas contribuições para a sustentabilidade: Proposição de um Sistema de Indicadores para Diagnóstico, Monitoramento e Avaliação. Campina Grande: UFCG, 2015.

ARAÚJO, R. O. A.; CÂNDIDO, G. A.. Sistema de Indicadores para Diagnóstico , Monitoramento e Avaliação de Tecnologias sociais: Proposição de uma metodologia. Revista Espacios, v.38, n.2, p.18, 2017

BAUER, A.. Avaliação de impacto no Brasil: É possível mensurar impactos de programas de formação docente?. Estudos em Avaliação Educacional, v.21, n 46, p.229-251, 2010.

CAVALCANTI, M.; NETO, A. P.. Inovação, tecnologias sociais e a política de ciência e tecnologia no Brasil: Desafio contemporâneo. 2011.

COELHO, M. Q.. Indicadores de performance para projetos sociais: a perspectiva dos stakeholders. Revista Alcance, v.11, n.3, p.423-444, 2004.

DAGNINO, R.. A tecnologia social e seus desafios Tecnologia Social, uma estratégia. 2004.

FERNANDES, R. M. C.; MACIEL, A. L. S.. Tecnologias Sociais: experiências e contribuições para o desenvolvimento social e sustentável. Porto Alegre: Fundação Irmão José Otão, 2010.

FONSECA, R.. Ciência, Tecnologia e Sociedade. In: TECNOLOGIA SOCIAL E DESENVOLVIMENTO SUSTENTÁVEL: CONTRIBUIÇÕES DA RTS PARA A FORMULAÇÃO DE UMA POLITICA DE ESTADO DE CIÊNCIA, TECNOLOGIA E INFORMAÇÃO. Anais. Brasília: RTS, 2010. p.71-78.

ITS. Instituto de Tecnologia Social. Caderno de debate: Tecnologia Social no Brasil. Brasília: 2004.
LASSANCE, A. E.; PEDREIRA, J. S.. Tecnologias sociais e políticas públicas. In: BRASIL, F. B.. Tecnologia social: uma estratégia para o desenvolvimento. 2004. p.65-83.

LAZZARINI, S. G.; PONGELUPPE, L. S.; YOONG, P. S.; ITO, N. C.. Guia para a Avaliação de Impacto Socioambiental para Utilização em Investimento de Impacto. Tac, v.5, n.2, p.10118, 2015.

MINAYO, M. C. S.. Construção de indicadores qualitativos para avaliação de mudanças. Revista Brasileira de Educação Médica, v.33, n.1, p.83-91, 2009.

OECD. Os Princípios da OCDE sobre o Governo das Sociedades. Ocde, 2004.

OLIVEIRA, D. B. S.. O Uso das Tecnologias Sociais Hídricas na Zona Rural do Semiárido Paraibano: entre o combate à seca e a convivência com o semiárido. João Pessoa: UFPB, 2013.

OLIVEIRA, N. D. A.; SILVA, T. N.. Inovação social e tecnologias sociais sustentáveis em relacionamentos intercooperativos: um estudo exploratório no CREDITAG-RO. Revista de Administração da UFSM, v.5, n.2, 2012.

SILVA, J. B.; GUERRA, L. D.; IORIS, A. A. R.; FERNANDES, M.. A crise hídrica global e as propostas do Banco Mundial e da ONU para seu enfrentamento. Revista Cronos, v.11, n.2, 2012.

STEPHANOU, M. C.. Análise Comparativa das Metodologias de Avaliação das Agências de Fomento Internacionais BID e BIRD em Financiamentos de Projetos Sociais no Brasil.

Civitas, v.5, n.1, p.127-160, 2005.

THOMAS, H. E.. Tecnologias para Inclusão Social e Políticas Públicas na América Latina. In: TECNOLOGIAS SOCIAIS: CAMINHOS PARA SUSTENTABILIDADE. Anais. Brasília: RTS, 2009. P.25-82.

VALARELLI, L. L.. Indicadores de resultados de projetos sociais. 1999.

A CBPC - Companhia Brasileira de Produção Científica (CNPJ: 11.221.422/0001-03) detém os direitos materiais desta publicação. Os direitos referem-se à publicação do trabalho em qualquer parte do mundo, incluindo os direitos às renovações, expansões e disseminações da contribuição, bem como outros direitos subsidiários. Todos os trabalhos publicados eletronicamente poderão posteriormente ser publicados em coletâneas impressas sob coordenação da Sustenere Publishing, da Companhia Brasileira de Produção Científica e seus parceiros autorizados. Os (as) autores (as) preservam os direitos autorais, mas não têm permissão para a publicação da contribuição em outro meio, impresso ou digital, em português ou em tradução. 